\title{
The skeleton and musculature on foetal MRI
}

\author{
Ursula Nemec • Stefan F. Nemec • Deborah Krakow • \\ Peter C. Brugger • Gustavo Malinger • \\ John M. Graham Jr. • David L. Rimoin • Daniela Prayer
}

Received: 19 October 2010 /Revised: 21 November 2010 / Accepted: 27 January 2011 /Published online: 19 February 2011

(C) European Society of Radiology 2011

\begin{abstract}
Background Magnetic resonance imaging (MRI) is used as an adjunct to ultrasound in prenatal imaging, the latter being the standard technique in obstetrical medicine.

Methods Initial results demonstrate the ability to visualise the foetal skeleton and muscles on MRI, and highlight the potentially useful applications for foetal MRI, which has significantly profited from innovations in sequence technology. Echoplanar imaging, thick-slab T2-weighted (w) imaging, and dynamic sequences are techniques complementary to classical T2-w imaging.
\end{abstract}

\section{U. Nemec $\cdot$ D. Prayer}

Department of Radiology,

Division of Neuroradiology and Musculoskeletal Radiology,

Medical University Vienna,

Waehringer Guertel 18-20,

1090 Vienna, Austria

S. F. Nemec $(\bowtie) \cdot$ J. M. Graham Jr. • D. L. Rimoin Medical Genetics Institute, Cedars Sinai Medical Center, 8700 Beverly Boulevard, PACT Suite 400,

Los Angeles, CA 90048, USA

e-mail: stefan.nemec@cshs.org

\section{Krakow}

Department of Orthopedic Surgery,

David Geffen School of Medicine, UCLA,

Los Angeles, CA 90095, USA

\section{P. C. Brugger}

Center of Anatomy and Cell Biology,

Integrative Morphology Group, Medical University Vienna,

Waehringerstrasse 13,

1090 Vienna, Austria

G. Malinger

Fetal Neurology Clinic, Prenatal Diagnosis Unit,

Department of Obstetrics and Gynecology, Tel Aviv University,

Tel Aviv, Israel
Results Recent study data indicate that foetal MRI may be useful in the imaging of spinal dysraphism and in differentiating between isolated and complex skeletal deformities with associated congenital malformations, which might have an impact on pre- and postnatal management.

Conclusion More research and technical refinement will be necessary to investigate normal human skeletal development and to identify MR imaging characteristics of skeletal abnormalities.

Keywords Foetal MRI · Skeleton · Muscles · Congenital abnormalities

\section{Introduction}

Foetal magnetic resonance imaging (MRI) has become an increasingly used imaging technique, serving as an adjunct to ultrasound in prenatal diagnosis [1-4]. Numerous recent studies have emphasised the benefits of MRI for the demonstration of congenital abnormalities of the brain and lungs, or complex syndromes [1-7].

However, there are almost no reports in the literature describing MRI for the prenatal visualisation and diagnosis of skeletal and muscular abnormalities, with the exception of spinal dysraphism [8-10]. Therefore, the authors sought to demonstrate and summarise their preliminary results for the visualisation of the skeleton in utero on MRI. The imaging techniques are described in detail, and the potential impact of a prenatal MRI diagnosis is discussed.

\section{Imaging}

In addition to commonly used T2-weighted (w) imaging to depict foetal anatomy and anomalies at all gestational ages, 
Table 1 Application of T2-w single-shot (SSh) turbospin-echo (TSE) sequences

\begin{tabular}{ll}
\hline Parameters & Axial, coronal, and sagittal planes; repetition time (TR): shortest; echo time (TE): \\
& 100 ms; TSE factor: 92; field of view: $200-230 \mathrm{~mm}$; matrix: $256 \times 153$; slice \\
& thickness: 3-4 mm; slices: 18; flip angle: $90^{\circ}$; duration: $18.7 \mathrm{~s}$ \\
Assessment & Whole body evaluation \\
Clinical application & Head size and shape (microcephaly; encephaloceles) \\
& Brain (associated malformations; acquired anomalies) (TE: $140 \mathrm{~ms}$ in GW 18-29) \\
& Face and foetal profile (facial clefts) \\
& Orbits (content; interorbital distance) \\
& Teeth buds and hard palate (cleft palate) \\
& External ear size, shape, and position; and fluid-filled inner ears \\
& Thorax shape and lung volume (lung hypoplasia in skeletal dysplasias) \\
& Spine (content/extent of spinal dysraphism) \\
& Arms and legs; hands and feet (size, shape, number of fingers/toes) \\
& Musculature (decreased thickness; fatty atrophy after GW 30)
\end{tabular}

MRI protocols should include imaging techniques such as echo planar imaging (EPI), thick-slab T2-w sequences, and dynamic steady-state free precession (SSFP) sequences to visualise the developing musculoskeletal system $[1,11,12]$.

Tables 1, 2, 3 and 4 show the areas of application of these MRI sequences and feature the sequence parameters used in our institution.

\section{EPI}

Currently, ultrasound provides information about bone ossification in prenatal standard imaging. Ossification centres, visualised as areas of increased echogenicity, are evaluated with ultrasound from 9 gestational weeks onwards [13-15]. The paradigm of MRI as "virtually blind to bones" [16],

Table 2 Application of single-shot fast-field-echo (SSh FFE) sequences (echo-planar imaging)

\begin{tabular}{ll}
\hline Parameters & Coronal and sagittal planes; repetition time \\
& (TR): $3,000 \mathrm{~ms}$; echo time (TE): shortest; \\
& field of view: $230 \mathrm{~mm}$; matrix: $160 \times 95 ;$ \\
& slice thickness: $4 \mathrm{~mm}$; flip \\
& angle: $90^{\circ}$; duration: $12 \mathrm{~s}$ \\
Assessment & Foetal skeleton \\
Clinical application & Facial profile (after GW 28; delineation \\
& enhanced because of chemical shift by \\
& subcutaneous fat) \\
& Hard palate (cleft palate) \\
& Imaging of normal hyperintense cartilaginous \\
& epiphysis and hypointense diaphysis \\
& Bone length and shape (bent bones; \\
& skeletal dysplasias) \\
Ossification disorders (osteogenesis imperfecta)
\end{tabular}

which is the case with conventional T2-w imaging, is changing. Recent developments in sequence technology have overcome these previous deficiencies. EP sequences now provide the delineation of bony structures, depending on the gestational age. Currently, EPI represents the only technique by which to image the skeleton before 27 gestational weeks (GW) on MRI, as the bones are demonstrated as hypointense structures and the cartilaginous epiphyses as hyperintense structures (Fig. 1, Table 2) [1, 9, 11]. EPI shows a pronounced distinction between bone and adjacent musculature, but low spatial resolution limits the use of this technique [17]. Sequence parameters must be modified in older foetuses, as the distinction between bone and muscles becomes less conspicuous with advancing gestational age.

Table 3 Application of thick-slab T2-w imaging

\begin{tabular}{ll}
\hline Parameters & Coronal and sagittal planes; repetition time (TR): \\
& $8,000 \mathrm{~ms}$; echo time (TE): $400-800 \mathrm{~ms}$; field of \\
& view: $210-320 \mathrm{~mm}$; matrix: $256 \times 205$; slice \\
& thickness: $30-50 \mathrm{~mm}$; flip angle: $90^{\circ}$; up to 15 \\
& projections $\left(12^{\circ}-15^{\circ}\right.$ angulation); duration: $8 \mathrm{~s}$ \\
Assessment & Foetal proportions and surface, whole foetus \\
& from different angles \\
Clinical & Facial features (micrognathia; dysmorphic features) \\
application & Intrauterine growth restriction (IUGR) \\
& Dwarfism, mesomelia or rizomelia (skeletal dysplasias) \\
& Extremity positioning (contractures, arthrogryposis) \\
& Extremity thickness (hydrops; subcutaneous \\
& oedema; muscle mass) \\
& Limb deformity/deficiency (amniotic bands; \\
& clubfeet; skeletal dysplasias) \\
& External masses (extensive lymphangiomas) \\
& Discontinuity of the body surface (omphalocele; \\
& spina bifida)
\end{tabular}


Table 4 Application of dynamic steady-state free precession (SSFP) sequences

\begin{tabular}{ll}
\hline Parameters & Repetition time (TR): $3.14 \mathrm{~ms}$; echo time (TE): $1.57 \mathrm{~ms}$; field of view: $320 \mathrm{~mm} ;$ \\
& matrix: $176 \times 110$; slice thickness: $30 \mathrm{~mm}$; gap: 0; flip angle: $60^{\circ} ; 4-6$ frames per \\
& second; up to 8 repetitions; duration: $34 \mathrm{~s}$ \\
Assessment & Foetal movements \\
Clinical application & Imaging of movement patterns of extremities, head, and body; swallowing; and \\
& diaphragm excursions \\
& Contractures/arthrogryposis \\
& Akinesia (foetal akinesia deformation sequence; neuromuscular disorders) \\
& Foetal bulk motion
\end{tabular}

\section{Thick-slab T2-w sequences}

Thick-slab T2-w imaging generates a three-dimensional (3D) image of the foetus (Fig. 2, Table 3). The whole foetus is covered by this technique, even in the third trimester, because of a large field of view (FOV) [12], which cannot be supplied by ultrasound. Abnormal proportions and some anomalies may be more easily recognised on a single image, which provides an overview of the whole foetus [12, $18,19]$. Thus, thick-slab T2-w sequences may be useful in cases of clubfeet, focal malpositioning disorders, or arthrogryposis (Fig. 3) [12]. The visualisation of long bones and extremities on commonly used slices from multiplanar MRI sequences may be impaired by the lack of limb continuity. Thick-slab images depict the shape and position of the whole limb on one image. This information may be crucial in cases of limb deformation or deficiency for any reason. The thick-slab $\mathrm{T} 2-\mathrm{w}$ method provides a "shine-through" effect for the inner foetal organs and may represent an alternative to 3D US in certain cases (Fig. 2) $[12,18,19]$.

\section{Dynamic sequences}

Ultrasound allows real-time visualisation of any plane in foetal imaging, and is, therefore, a true dynamic imaging method, contrary to MRI $[20,21]$. The presence, reduction, or absence of foetal movements of the extremities can be better delineated on ultrasound than on foetal MRI [1]. However, the introduction of dynamic SSFP sequences using four to six images per second has permitted the demonstration of movements of the foetal extremities and gross foetal motion (Fig. 4) [1, 11, 22]. MRI may not cover the extremities or even detect any foetal details if the foetus is rapidly moving, which can be overcome with dynamic sequences (Table 4) [11,23]. Some limb abnormalities will be more obvious, in motion, on dynamic images. Therefore, dynamic MR images are instrumental for the evaluation of extremity malformations, as well as movement disorders. Quantitative movement studies are not performed with MRI [1]. Consequently, the examining physician should be cautious about diagnosing movement reduction on 30-60-s MR studies. The movement pattern of the extremities should
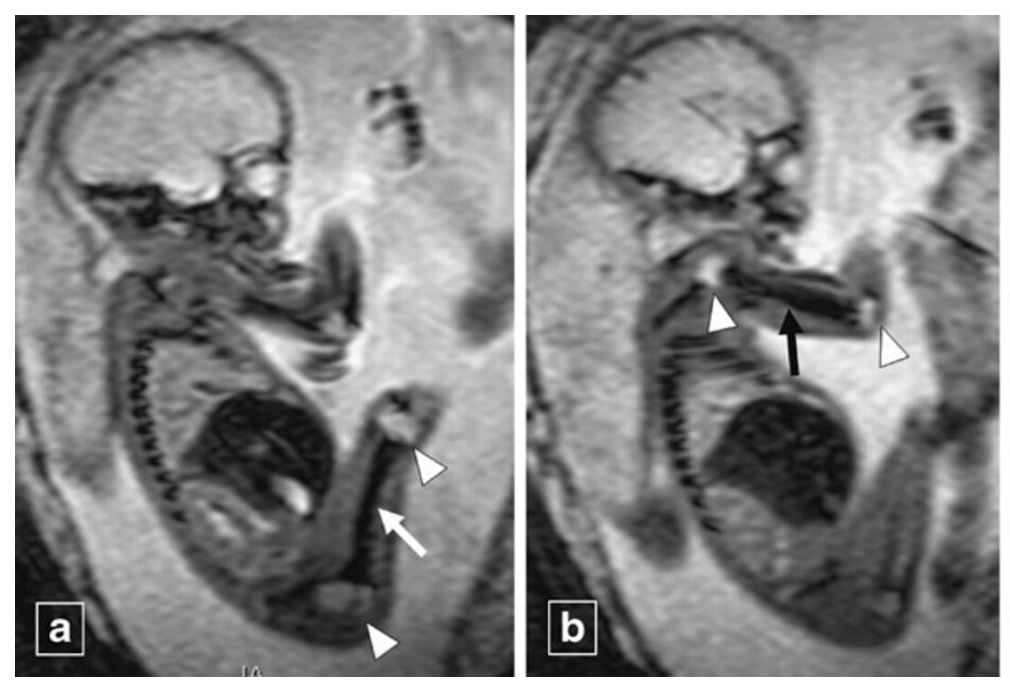

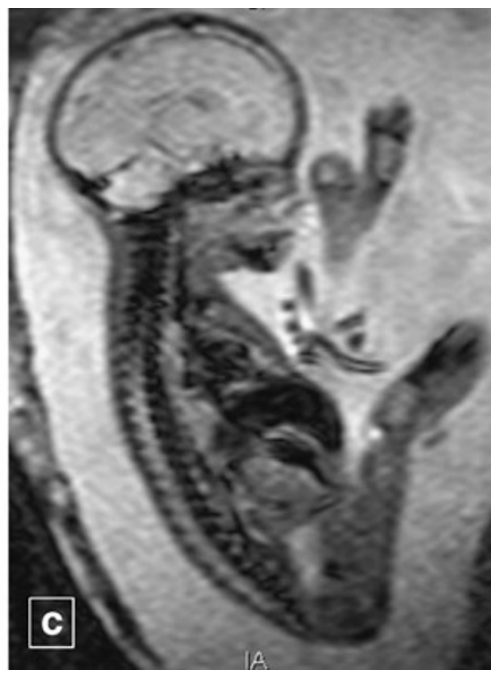

Fig. 1 Foetus at $24+2$ gestational weeks (GW) with normal skeletal development. The sagittal echo planar (EP) images demonstrate the femur (a) and the humerus (b) with hypointense diaphysis (arrows) and hyperintense proximal and distal epiphysis (arrowheads); normal hypointense ossification of the spine according to the gestational age (c) 


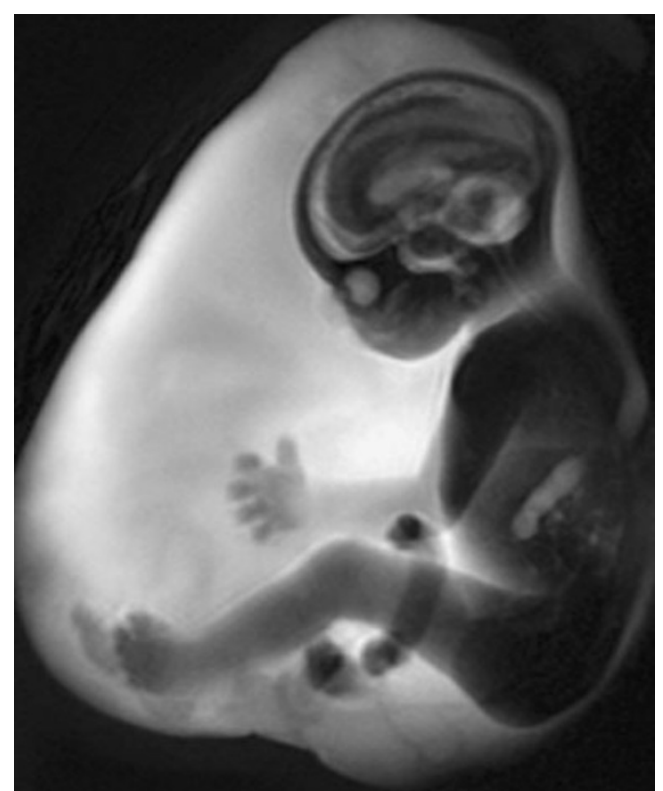

Fig. 2 Foetus at $33+0 \mathrm{GW}$ with normal anatomy in polyhydramnios. The thick-slab T2-w sequence enables the visualisation of the whole foetus on one image and shows normal positioning of the extremities

be assessed based on published ultrasound criteria [21] in dynamic sequences, applied five times, at about 5, 10, 15, 20, and $25 \mathrm{~min}$. A modification of the ultrasound criteria [21] (reduced amplitude and speed, or number of participating body parts) may be used to evaluate generalised disorders or focal mobility problems. The fixed nature of an extremity contracture can be visualised as the absence of changes in the extremity's position during the course of the examination. Dynamic MRI studies should be evaluated only in combination with morphology to differentiate a possible temporary variant of malpositioning from an actual abnormality that may affect the musculoskeletal system or the nervous system, particularly the brainstem. Foetal akinesia, defined by an absence of motion, could be interpreted as a sign of (neuro) muscular disease in combination with thin muscles and polyhydramnios (Fig. 5); lung hypoplasia and certain facial abnormalities could suggest a Pena-Shokeir phenotype [24].

\section{Skull}

The size and shape of the foetal head/skull is usually visualised on ultrasound studies [25]. In the case of asymmetrical head enlargement, MRI may be useful in excluding the presence of associated cerebral malformations $[1,26]$. Possible encephaloceles should be examined for their content (Fig. 6). Both 2D and 3D ultrasound provides detailed visualisation of cleft lip and the anterior palate [27]. The examination of the posterior palate on ultrasound may be impaired due to acoustic bony shadowing [27]. In comparison, T2-w MRI demonstrates the posterior palate consistently. MRI has been used for the detection of isolated cleft palate, as well as clefts of both the primary and secondary palate (Fig. 7) [28]. Isolated cleft lip, however, may be impossible to diagnose on MRI because of partial volume averaging (Tables 1,2).

On MRI, the skull base and cranio-cervical junction should be examined in detail, particularly the width and content of the foramen magnum, as a narrow foramen magnum may occur in some skeletal dysplasias, and cerebellar herniation occurs in Chiari malformations [10].

\section{Thorax and spine}

Although MRI can visualise the shape of the thorax, which may be essential in some syndromes or skeletal dysplasias (Fig. 8), its predominant task is the assessment of the foetal lungs $[1,5]$. Concerning the foetal spine, results indicate that MRI may provide additional information beyond ultrasound in foetuses with spina bifida and subsequent neurosurgery [15]. Based upon advanced resolution, this type of spinal dysraphism can be better evaluated on MRI than on ultrasound, as MRI provides an exact delineation of the cord and the interface between cerebrospinal fluid and the extradural space (Table 1) [15]. The prognosis of spinal dysraphism is influenced by the level and the type of the lesion, the possibility of associated anomalies, ventriculomegaly, and the type of closure. The options for a pregnant woman in the case of confirmed spina bifida include termination of pregnancy, foetal surgery, and planned Caesarean section with immediate postnatal surgical repair. Overall, MRI has been recommended for preoperative

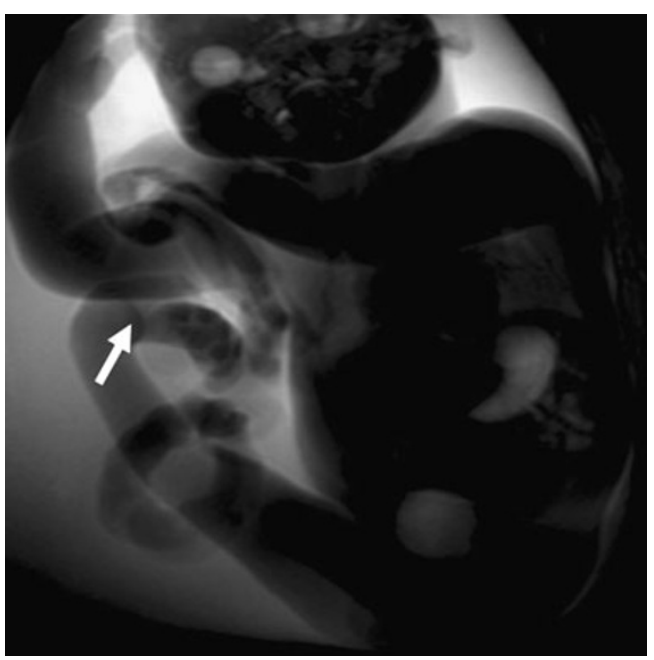

Fig. 3 Foetus at 31+2 GW with multiple contractures. The thick-slab T2-w image shows an abnormal inward rotation of the foot compared with the course of the calf (arrow), resulting in a clubfoot deformity 

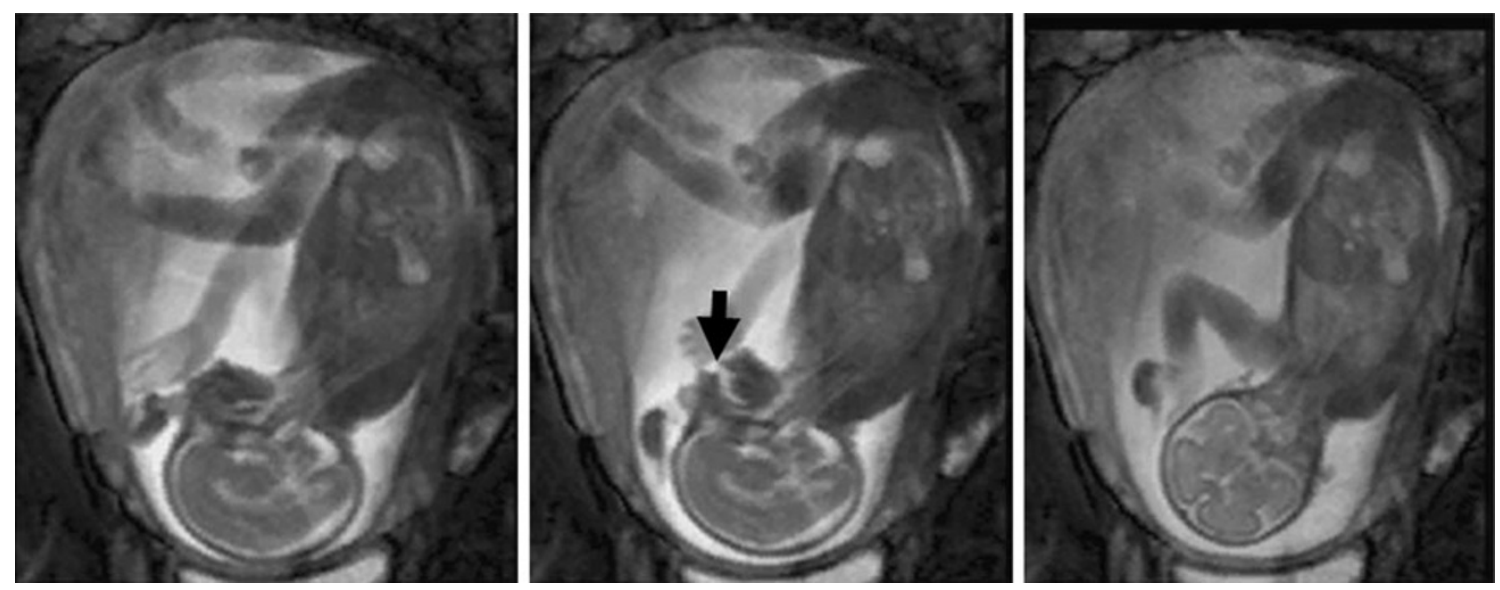

Fig. 4 Foetus at 27+4 GW with normal motor development. The sequence of dynamic images (from left to right) shows active movement of the extremities, the body, and the head, as well as jaw movements (arrow) and swallowing

evaluation of spinal dysraphism, particularly for foetuses with an open spinal canal and associated cerebral or cerebellar malformations (Fig. 9) [8, 10]. Congenital scoliosis, as a result of improper formation and/or segmentation, is the most frequent congenital deformity of the spine [29]. MRI may be helpful in the diagnosis of malformations or other anomalies often associated with this condition (Fig. 10).

\section{Long bones and extremities}

Ultrasound is the method of choice for measuring long bones and observing subtle findings that involve the distal appendages, which can also be observed with foetal MRI $[30,31]$. The extremities are particularly well suited to imaging with 3D ultrasound [30, 31]. However, it may be difficult or impossible to assess all extremities sonographically on the dependent side of the foetus, or if the amniotic fluid is severely reduced, or late in gestation. On MRI, EPI sequences can be used to image foetal bone and skeletal development. Details of the developing epiphysis may be recognised (Fig. 11) [1, 11], and disorders of ossification may be visualised (Fig. 12, Table 2). Connolly et al. described the MR imaging appearances of the foetal pig femur at each trimester and correlated the results with histology [32]. In that study, MRI depicted contour changes of the femoral intercondylar notch, changes in the epiphyseal ossification centre, and changes in the signal intensity and shape of the bone marrow [32]. Currently, the stages of bone development on MRI are not entirely known for human foetuses, and there is only initial experience in the visual-
Fig. 5 Foetus at $27+1 \mathrm{GW}$ with neuromuscular disease. The thick-slab T2-w image shows the foetus with generalised subcutaneous oedema, thin extremities, and polyhydramnios (a). The axial T2-w image of the thorax and upper extremities demonstrates, in addition to diffuse oedema, partial atrophy of the musculature (arrow) (b)
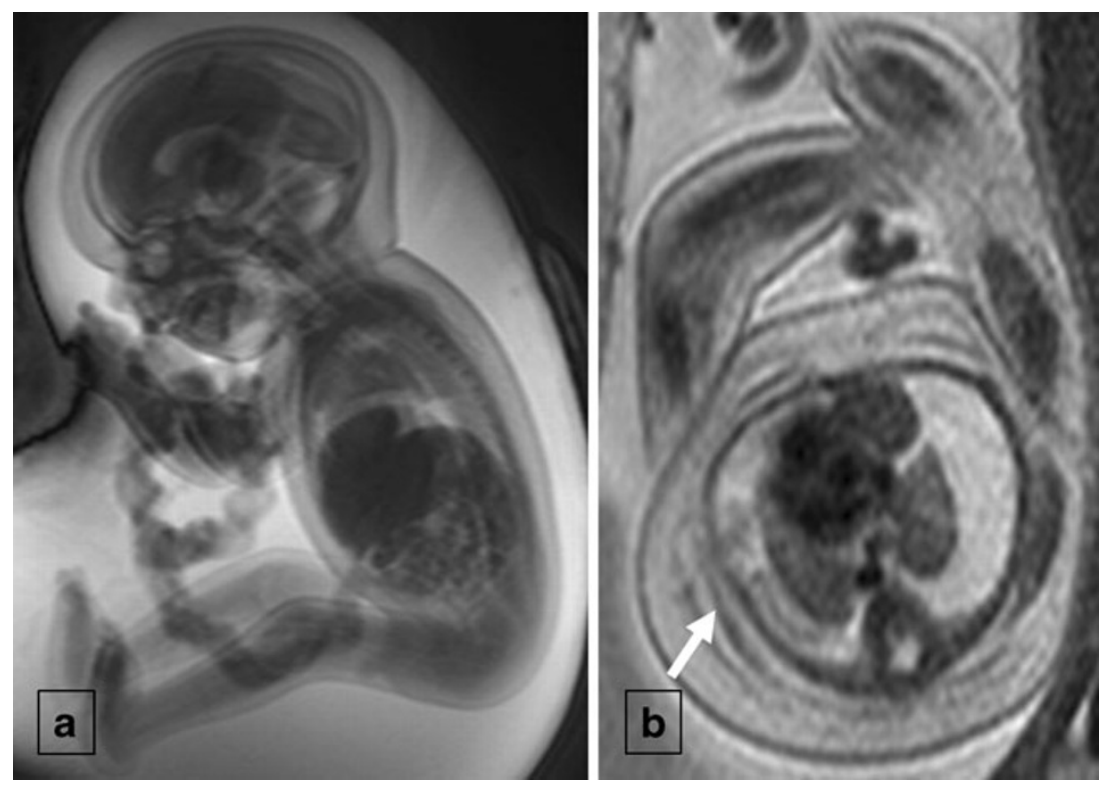


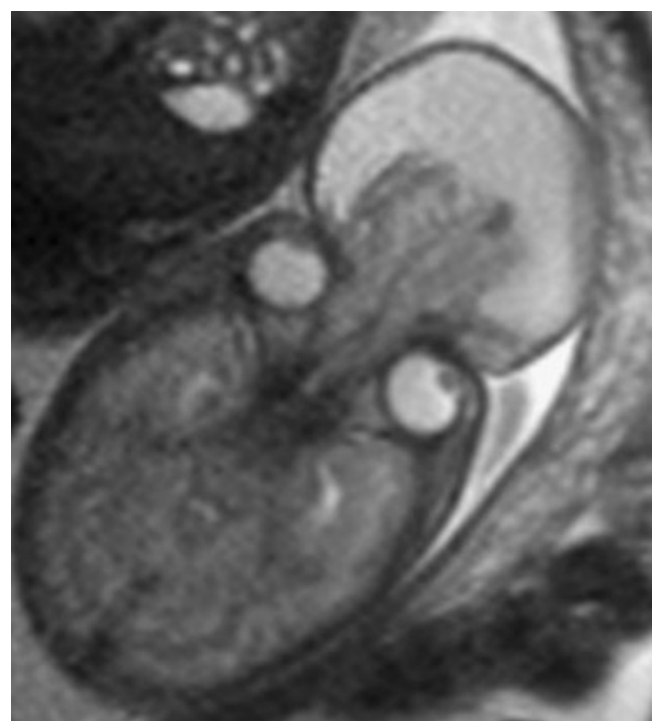

Fig. 6 Foetus at $32+2 \mathrm{GW}$ with craniofacial maldevelopment. The axial T2-w image of the head shows a large orbitofrontal meningoencephalocele, with distinct protrusion of the frontal lobes

isation of foetal bones [33]. In growth-restricted foetuses, the MRI appearance of the placenta may provide an indication of the severity and underlying disease process [34].

\section{Muscles}

Magnetic resonance imaging is the most accurate method of delineating any kind of muscular abnormalities in paediatric

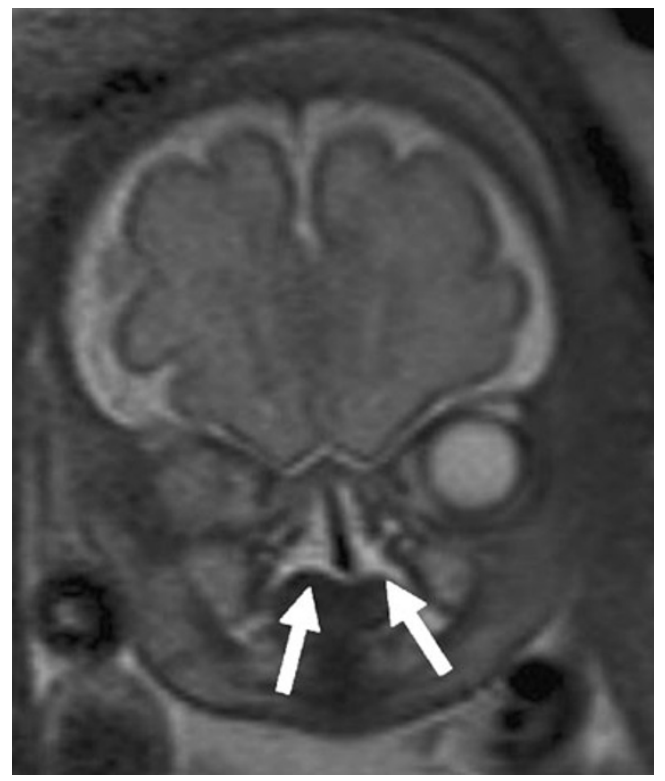

Fig. 7 Foetus at $30+0 \mathrm{GW}$ with bilateral cleft lip and palate and otherwise normal anatomy. The coronal T2-w image of the face shows a broad fluid (hyperintense) connection between the naso- and oropharynx on both sides (arrows)

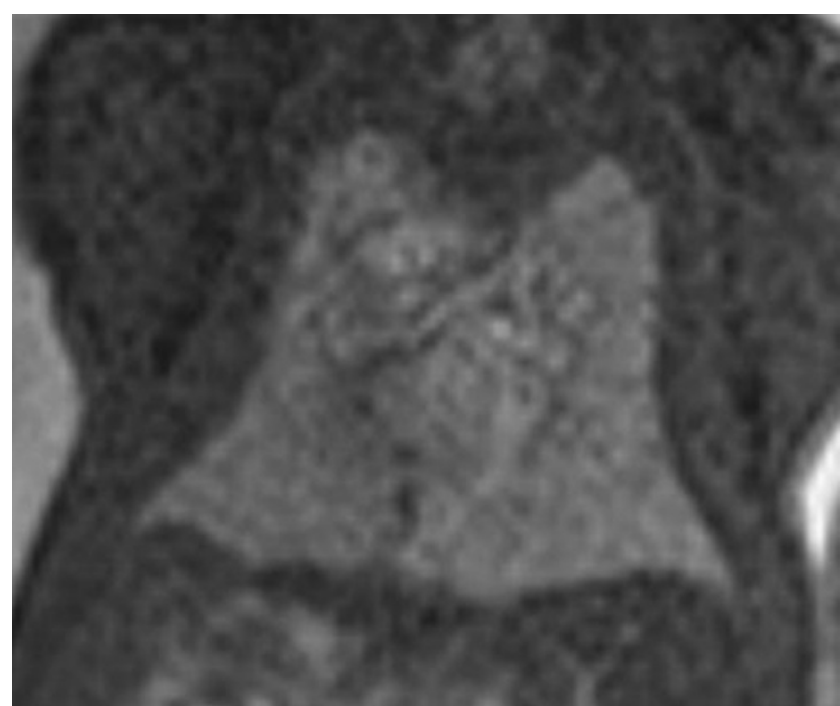

Fig. 8 Foetus at $32+0 \mathrm{GW}$ with thoracic deformity in unclassified syndrome. The coronal T2-w image shows abnormally shaped lungs, with narrowing of the mid-thorax due to rip deformities; the latter are not clearly visible on the image

populations [35], but MRI of the foetal musculature is only in the early stages of investigation. With regard to the structure, normal individual muscles (with few exceptions, e.g., the diaphragm) cannot be delineated, because muscles display a homogeneous T2-w hypointensity [1]. However, tissue composition changes with advancing gestation, as evidenced by EPI sequences. MRI can show the thickness and contours of the skeletal muscles, and atrophy (Fig. 5) [1], which may indicate the presence of a neuromuscular disorder. In addition, $\mathrm{T} 1-$ and $\mathrm{T} 2-\mathrm{w}$ signals may be pathological with increased T2-w signal intensities (Table 1) [1]. It has been stated that impairment of muscle development must reach a critical stage, occurring relatively late in pregnancy, to result in significant changes in foetal motility and morphology [36]. Overall, abnormal muscular development may be observed with limb abnormalities, such as arthrogryposis, spinal muscle dystrophy, or muscle dystrophy $[37,38]$.

\section{Complex defects of the musculoskeletal system}

Abnormalities may be either isolated or may be accompanied by other defects, particularly in genetic disorders or in chromosomal syndromes [39]. The prenatal diagnosis of musculoskeletal anomalies should be based on information assembled from imaging combined with biochemical and genetic workups [40, 41]. Prenatal diagnosis will serve as a prognostic tool and in counselling the parents. The management of pregnancies with musculoskeletal disease depends mainly on three factors: the time of diagnosis, the severity of disease, and the parents' 
Fig. 9 Foetus at $23+3 \mathrm{GW}$ with a Chiari II malformation. (a) The sagittal T2-w image of the whole foetus shows a lumbosacral neural tube defect (ellipsis) and a herniation of the cerebellum (arrow). The sagittal (b) and axial (c) T2-w images of the body and pelvis show a large spinal defect referring to a meningomyelocele that extends from the level of L2 to the end of the sacrum (ellipsis). The axial T2-w image of the brain demonstrates internal hydrocephaly with marked widening of the lateral ventricles (d)
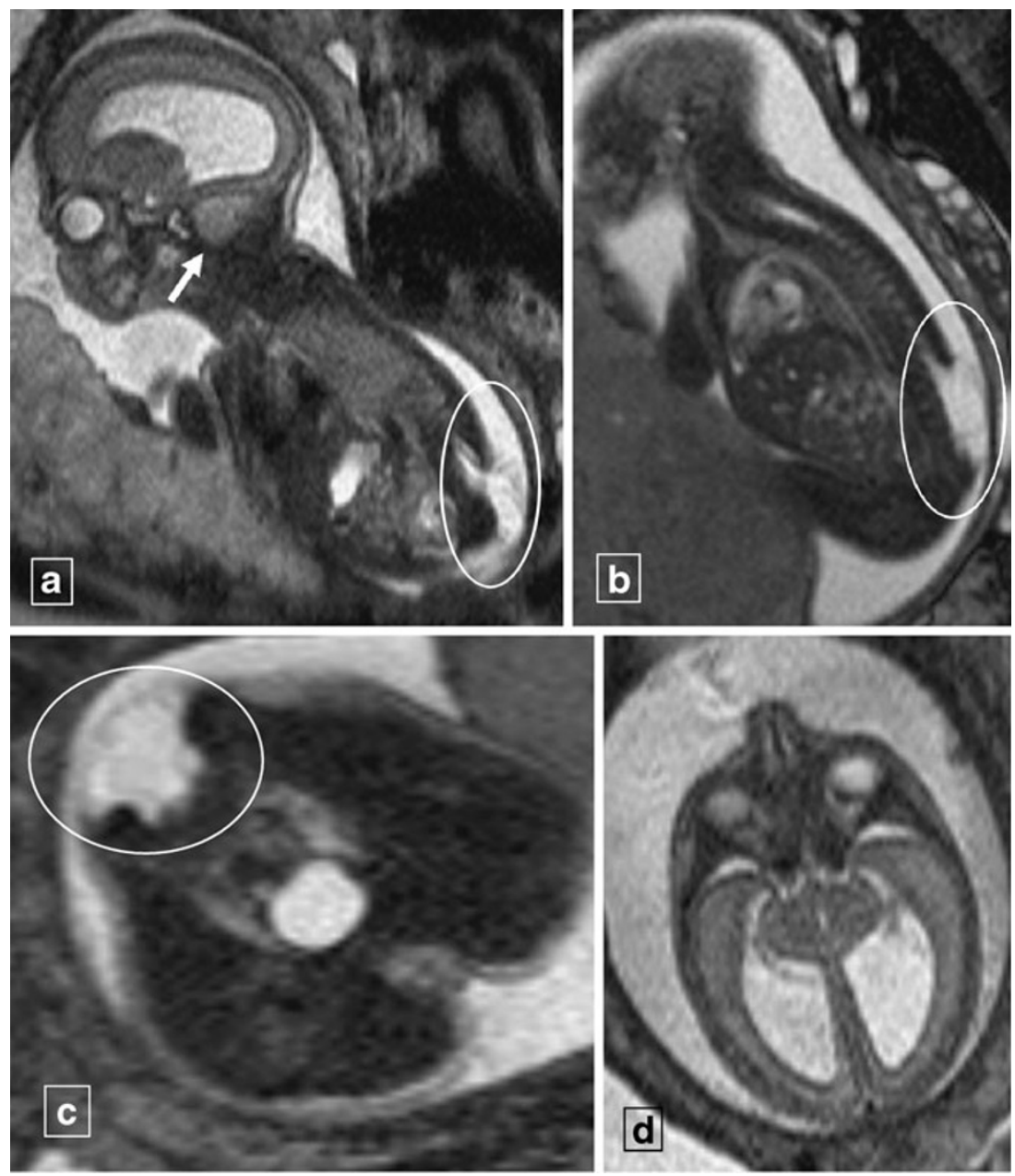

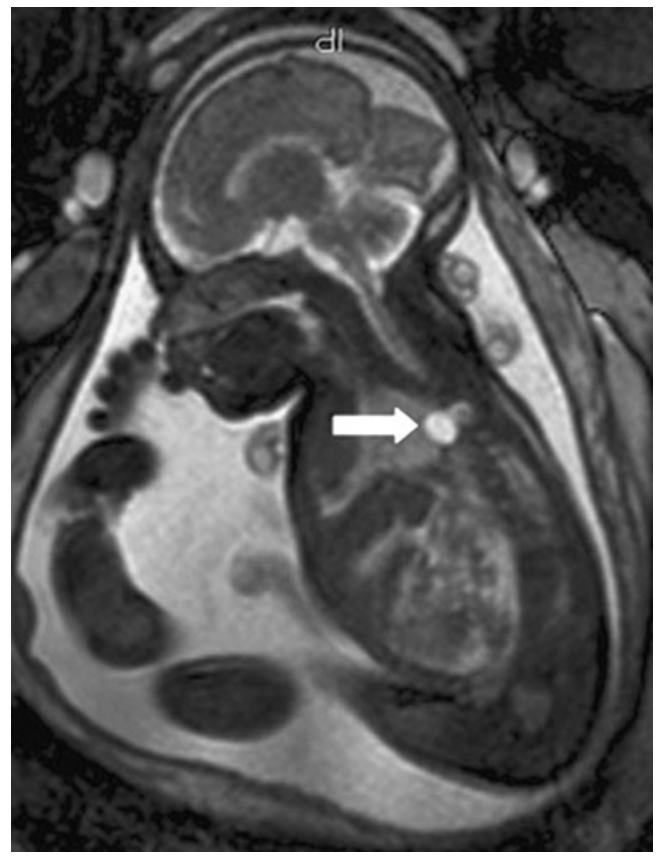

Fig. 10 Foetus at 29+1 GW with scoliosis. The sagittal T2-w image of the whole foetus demonstrates a paravertebral cystic lesion referred to as an intrathoracic neurenteric cyst (arrow) decision [15]. When identifying a musculoskeletal abnormality, the whole foetus should be assessed carefully, particularly the CNS and the lungs, to detect any abnormalities (Fig. 13) [42]. The presence or absence of specific MRI findings will help to differentiate between isolated and complex anomalies. For instance, amniotic bands should be considered in cases of isolated limb deficiency [43]. Figure 14 features a mnemonic about the most important imaging considerations when imaging the extremities.

Foetal skeletal dysplasias are usually recognised by ultrasound [41, 44-46]. However, published reports have described ultrasound as only moderately accurate in the detection of foetal musculoskeletal anomalies and specific skeletal dysplasias [45, 46], which may indicate a potential future role of MRI in these conditions. Foetal MR imaging may complement the use of molecular genetics to diagnose skeletal dysplasias [33]. After 30 weeks of gestation, conventional radiography of the maternal abdomen may also help to identify possible bone abnormalities [45]. Recent case series report the application of prenatal computed tomography with $3 \mathrm{D}$ reconstructions as an 

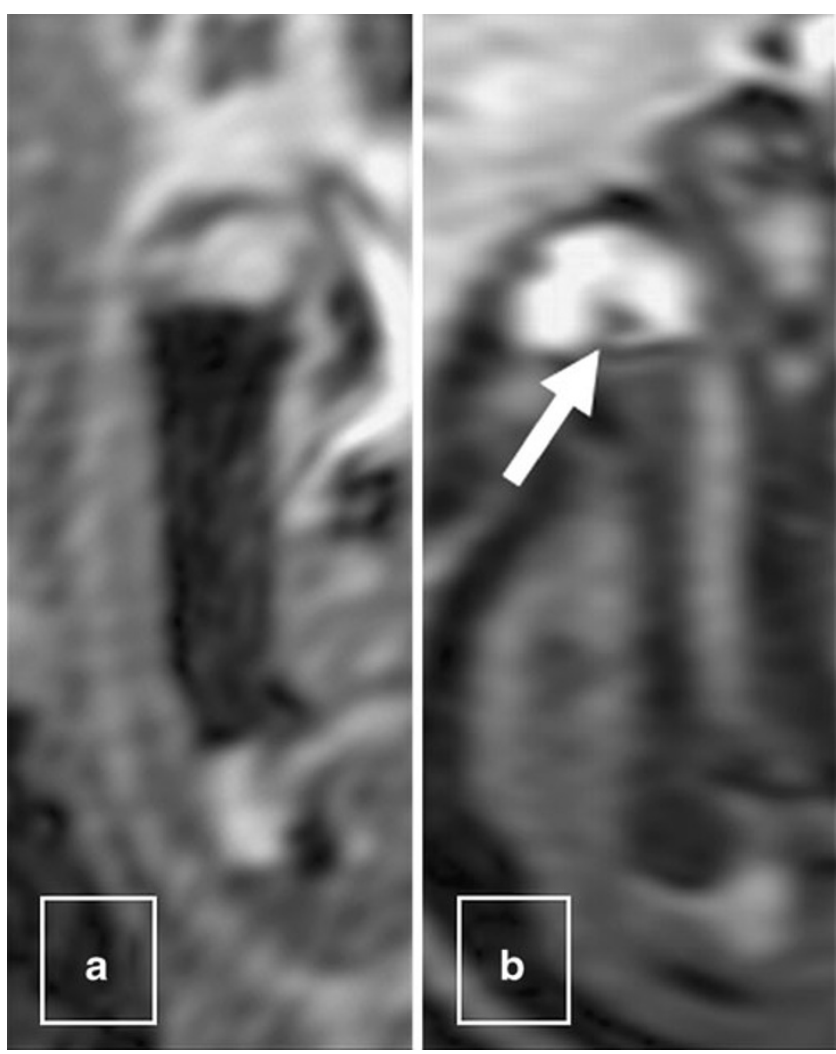

Fig. 11 Foetuses with normal long-bone development at 24 and 40 GW. The sagittal EP images of the thigh demonstrate hyperintense epiphyseal structures, with no distal femoral ossification centre at 24 GW (a), and a small hypointense ossification centre (arrow) in the older foetus (b)

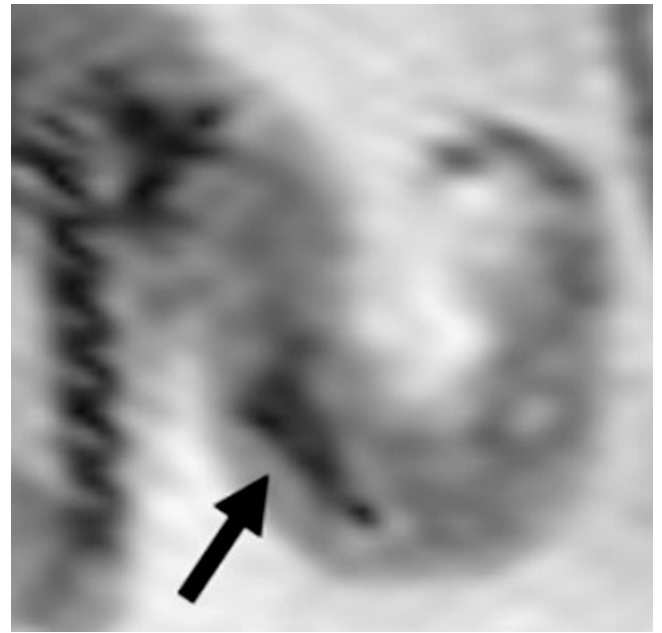

Fig. 12 Foetus at $22+6 \mathrm{GW}$ with lethal hypophosphatasia. The coronal EP image of the upper extremity shows a short and abnormally shaped humerus (arrow) and no ossification of the bones of the forearm, which was indicative of the mineralisation disorder

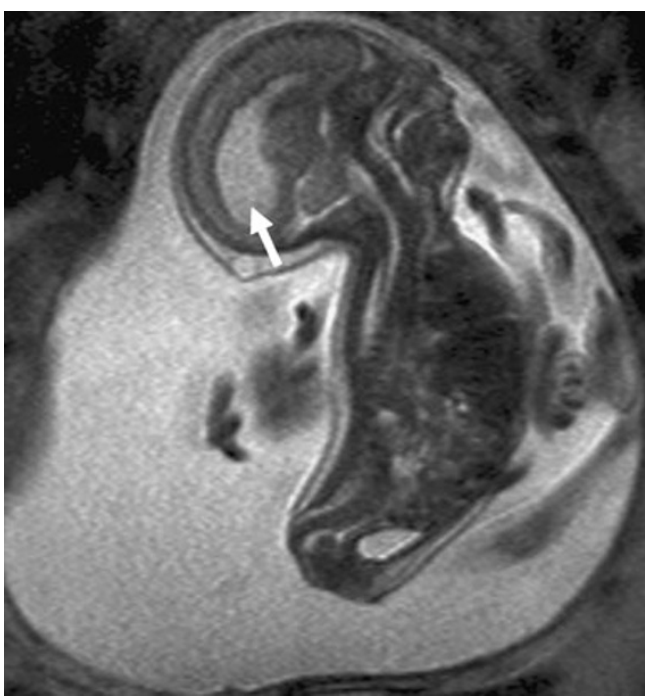

Fig. 13 Foetus at $21+6 \mathrm{GW}$ with complex musculoskeletal abnormalities and foetal akinesia in polyhydramnios. The sagittal T2-w image of the whole foetus shows a short malformed spine with severe scoliosis. The skull is also elongated, referring to dolichocephaly with internal hydrocephaly (arrow)

adjunct to ultrasound in the diagnosis of lethal skeletal dysplasias [47].

\section{Conclusion}

Initial results demonstrate the visualisation of the normal and abnormal skeleton on foetal MRI, which highlights the use of MRI as an adjunct to prenatal ultrasound. MRI may be helpful in foetal spinal imaging, and in the differentiation between isolated and complex abnormalities, which might have an impact on pre- and postnatal management, as complex abnormalities may be related to an unfavourable outcome. Preliminary experience suggests an improvement in diagnosis through additional MRI findings in specific cases compared with ultrasound. However, increased efforts are needed to refine MRI techniques for the visualisation of the foetal skeleton and to clarify the value of MRI compared with standard ultrasound.

\section{Size and shape Isolated or complex pathologies Muscles \\ Bones (number; signal intensity) Abnormal movement}

Fig. 14 Mnemonic for MRI 
Acknowledgments U.N. and S.F.N. co-wrote the manuscript; both authors contributed equally to this work. The authors would like to thank Ms. Mary McAllister for her help in editing the manuscript.

Financial disclosure The authors have indicated they have no financial relationships relevant to this article to disclose.

\section{Conflict of interest None}

\section{References}

1. Prayer D, Brugger PC (2007) Investigation of normal organ development with fetal MRI. Eur Radiol 17:2458-2471

2. Breysem L, Bosmans H, Dymarkowski S et al (2003) The value of fast MR imaging as an adjunct to ultrasound in prenatal diagnosis. Eur Radiol 13:1538-1548

3. Garel C (2008) Imaging the fetus: when does MRI really help? Pediatr Radiol 38(Suppl 3):S467-S470

4. Garel C (2008) Fetal MRI: what is the future? Ultrasound Obstet Gynecol 31:123-128

5. Balassy C, Kasprian G, Brugger PC, Weber M, Csapo B, Herold C, Prayer D (2010) Assessment of lung development in isolated congenital diaphragmatic hernia using signal intensity ratios on fetal MR imaging. Eur Radiol 20:829-837

6. Fogliarini C, Chaumoitre K, Chapon F et al (2005) Assessment of cortical maturation with prenatal MRI. Part I: Normal cortical maturation. Eur Radiol 15:1671-1685

7. Fogliarini C, Chaumoitre K, Chapon F et al (2005) Assessment of cortical maturation with prenatal MRI: part II: abnormalities of cortical maturation. Eur Radiol 15:1781-1789

8. Bulas D (2010) Fetal evaluation of spine dysraphism. Pediatr Radiol 40:1029-1037

9. Servaes S, Hernandez A, Gonzalez L et al (2010) Fetal MRI of clubfoot associated with myelomeningocele. Pediatr Radiol 40:1874-1879

10. Hüsler MR, Danzer E, Johnson MP et al (2009) Prenatal diagnosis and postnatal outcome of fetal spinal defects without ArnoldChiari II malformation. Prenat Diagn 29:1050-1057

11. Brugger PC, Stuhr F, Lindner C, Prayer D (2006) Methods of fetal MR: beyond T2-weighted imaging. Eur J Radiol 57:172-181

12. Brugger PC, Mittermayer C, Prayer D (2006) A new look at the fetus: thick-slab T2-weighted sequences in fetal MRI. Eur J Radiol 57:182-186

13. Donne HD Jr, Faúndes A, Tristão EG, de Sousa MH, Urbanetz AA (2005) Sonographic identification and measurement of the epiphyseal ossification centers as markers of fetal gestational age. J Clin Ultrasound 33:394-400

14. van Zalen-Sprock RM, Brons JT, van Vugt JM, van der Harten HJ, van Geijn HP (1997) Ultrasonographic and radiologic visualization of the developing embryonic skeleton. Ultrasound Obstet Gynecol 9:392-397

15. Blaicher W, Mittermayer C, Messerschmidt A, Deutinger J, Bernaschek G, Prayer D (2004) Fetal skeletal deformities - the diagnostic accuracy of prenatal ultrasonography and fetal magnetic resonance imaging. Ultraschall Med 25(3):195-199

16. Brunelle F (2001) Fetal imaging in a new era. Ultrasound Obstet Gynecol 18:91-95

17. Chen Q, Levine D (2001) Fast fetal magnetic resonance imaging techniques. Top Magn Reson Imaging 12:67-79

18. Huisman TA, Solopova A (2009) MR fetography using heavily T2-weighted sequences: comparison of thin- and thick-slab acquisitions. Eur J Radiol 71:557-563
19. Chaumoitre K, Wikberg E, Shojai R et al (2006) Fetal magnetic resonance hydrography: evaluation of a single-shot thick-slab RARE (rapid acquisition with relaxation enhancement) sequence in fetal thoracoabdominal pathology. Ultrasound Obstet Gynecol 27:537-544

20. de Vries JI, Fong BF (2006) Normal fetal motility: an overview. Ultrasound Obstet Gynecol 27:701-711

21. Lüchinger $A B$, Hadders-Algra $M$, van Kan CM, de Vries JI (2008) Fetal onset of general movements. Pediatr Res 63:191-195

22. Chung R, Kasprian G, Brugger PC, Prayer D (2009) The current state and future of fetal imaging. Clin Perinatol 36:685-699

23. Pugash D, Brugger PC, Bettelheim D, Prayer D (2008) Prenatal ultrasound and fetal MRI: the comparative value of each modality in prenatal diagnosis. Eur J Radiol 68:214-226

24. Senocak EU, Oguz KK, Haliloglu G, Karcaaltincaba D, Akata D, Kandemir O (2009) Prenatal diagnosis of Pena-Shokeir syndrome phenotype by ultrasonography and MR imaging. Pediatr Radiol 39:377-380

25. Rochelson B, Vohra N, Krantz D, Macri VJ (2006) Geometric morphometric analysis of shape outlines of the normal and abnormal fetal skull using three-dimensional sonographic multiplanar display. Ultrasound Obstet Gynecol 27:167-172

26. Glass RB, Fernbach SK, Norton KI, Choi PS, Naidich TP (2004) The infant skull: a vault of information. Radiographics 24:507522

27. Ramos GA, Romine LE, Gindes L et al (2010) Evaluation of the fetal secondary palate by 3-dimensional ultrasonography. J Ultrasound Med 29:357-364

28. Mailáth-Pokorny M, Worda C, Krampl-Bettelheim E, Watzinger F, Brugger P, Prayer D (2010) What does fetal MRI add to the prenatal ultrasound diagnosis of facial clefts? Ultrasound Obstet Gynecol 36:445-451

29. Arlet V, Odent T, Aebi M (2003) Congenital scoliosis. Eur Spine J 12:456-463

30. Paladini D, Greco E, Sglavo G, D'Armiento MR, Penner I, Nappi C (2010) Congenital anomalies of upper extremities: prenatal ultrasound diagnosis, significance, and outcome. Am J Obstet Gynecol 202:596.e1-10

31. Krakow D, Williams J 3rd, Poehl M, Rimoin DL, Platt LD (2003) Use of three-dimensional ultrasound imaging in the diagnosis of prenatal-onset skeletal dysplasias. Ultrasound Obstet Gynecol $21: 467-472$

32. Connolly SA, Jaramillo D, Hong JK, Shapiro F (2004) Skeletal development in fetal pig specimens: MR imaging of femur with histologic comparison. Radiology 233:505-514

33. Applegate KE (2004) Can MR imaging be used to characterize fetal musculoskeletal development? Radiology 233:305-306

34. Damodaram M, Story L, Eixarch E et al (2010) Placental MRI in intrauterine fetal growth restriction. Placenta 31:491-498

35. MacKenzie JD, Vasanawala SS (2010) State-of-the-art in pediatric body and musculoskeletal magnetic resonance imaging. Semin Ultrasound CT MR 31:86-99

36. Mulder EJ, Nikkels PG, Visser GH (2001) Fetal akinesia deformation sequence: behavioral development in a case of congenital myopathy. Ultrasound Obstet Gynecol 18:253-257

37. Bevan WP, Hall JG, Bamshad M, Staheli LT, Jaffe KM, Song K (2007) Arthrogryposis multiplex congenita (amyoplasia): an orthopaedic perspective. J Pediatr Orthop 27:594-600

38. Hall JG (2008) Pena-Shokeir phenotype (fetal akinesia deformation sequence) revisited. Birth Defects Res A Clin Mol Teratol 85:677-694

39. Lachman RS (2006) Taybi \& Lachman's radiology of syndromes, metabolic disorders and skeletal dysplasias, 5th edn. Mosby, Philadelphia

40. Krakow D, Rimoin DL (2010) The skeletal dysplasias. Genet Med $12: 327-341$ 
41. Krakow D, Lachman RS, Rimoin DL (2009) Guidelines for the prenatal diagnosis of fetal skeletal dysplasias. Genet Med 11:127133

42. Miller E, Blaser S, Shannon P, Widjaja E (2009) Brain and bone abnormalities of thanatophoric dwarfism. AJR Am J Roentgenol 192:48-51

43. Kawamura K, Chung KC (2009) Constriction band syndrome. Hand Clin 25:257-264

44. Krakow D, Alanay Y, Rimoin LP et al (2008) Evaluation of prenatal-onset osteochondrodysplasias by ultrasonography: a retrospective and prospective analysis. Am J Med Genet A 146A:1917-1924

45. Cassart M (2010) Suspected fetal skeletal malformations or bone diseases: how to explore. Pediatr Radiol 40:1046-1051

46. Schramm T, Gloning KP, Minderer S et al (2009) Prenatal sonographic diagnosis of skeletal dysplasias. Ultrasound Obstet Gynecol 34:160-170

47. Tsutsumi S, Sawai H, Nishimura G, Hayasaka K, Kurachi H (2008) Prenatal diagnosis of thanatophoric dysplasia by 3-D helical computed tomography and genetic analysis. Fetal Diagn Ther 24:420-424 